\title{
FRIDMAN'S INVARIANT, SQUEEZING FUNCTIONS, AND EXHAUSTING DOMAINS
}

\author{
FUSHENG DENG, XUJUN ZHANG
}

\begin{abstract}
We show that if a bounded domain $\Omega$ is exhausted by a bounded strictly pseudoconvex domain $D$ with $C^{2}$ boundary, then $\Omega$ is holomorphically equivalent to $D$ or the unit ball, and show that a bounded domain has to be holomorphically equivalent to the unit ball if its Fridman's invariant has certain growth condition near the boundary.
\end{abstract}

\section{INTRODUCTION}

In [18, Fridman introduces a new invariant for bounded domains as follows. Let $D \subset \mathbb{C}^{n}$ be a bounded domain and $z \in D$. Let $f: B^{n} \rightarrow D$ be a holomorphic injective map such that $f(0)=z$, where $B^{n}$ is the unit ball in $\mathbb{C}^{n}$. We define $q_{D, f}(z)$ to be the supremum of all $r>0$ such that $B_{D}^{K}(z, r) \subset f\left(B^{n}\right)$, where $B_{D}^{K}(z, r)$ is the open ball in $D$ centered at $z$ with radius $r$ with respect to the Kobayashi metric on $D$. Then Firdman's invariant is defined to be

$$
h_{D}(z)=\inf _{f} \frac{1}{q_{D, f}(z)},
$$

where the supremum is taken to be all injective holomorphic maps $f: B^{n} \rightarrow D$ with $f(z)=0$.

Form the definition, it is clear that $h_{D}$ is invariant under biholomorphic transformations. Some basic results about the invariant $h_{D}$ were proved in [18. The most important one says that for a bounded strictly pseudoconvex domain $D$ with $C^{3}$ boundary, $h_{D}(z)$ tends to 0 as $z$ goes to the boundary.

In [6, Deng-Guan-Zhang introduce another invariant of bounded domains, called squeezing function, as follows. Let $D$ be a bounded domain in $\mathbb{C}^{n}$ and $z \in D$. For an injective holomorphic map $f: D \rightarrow B^{n}$ with $f(z)=0$, we define

$$
s_{D}(p, f)=\sup \{r \mid B(0, r) \subset f(D)\},
$$

and define

$$
s_{D}(z)=\sup _{f}\left\{s_{D}(p, f)\right\},
$$

where the supremum is taken over all injective holomorphic maps $f: D \rightarrow B^{n}$ with $f(p)=0$, and $B^{n}(0, r)$ is the Euclidean ball in $\mathbb{C}^{n}$ with center 0 and radius $r$. As $z$ varies, we get a function $s_{D}$ on $D$, which is called the squeezing function of $D$.

By definition, a bounded domain is called homogenous regular if its squeezing function has positive lower bound. The notion of homogenous regular domains was introduced and studied in 22, 23, and was called uniform squeezing domains and systematically studied in 29 . 
In addition to the works in [22] 23] 29], the new motivation for introducing the concept of squeezing function is boundary estimate. The study of boundary estimate of squeezing functions was initiated in [6] and was further developed in [10] 7. In recent years, boundary estimate of squeezing functions and their applications in different settings were extensively studied by different authors (see e.g.

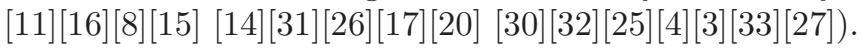

One motivation for Fidman to propose the invariant $h_{D}$ defined above is to study exhausting domains. Let $D, \Omega$ be domains in $\mathbb{C}^{n}$. Following Fridman, we say that $\Omega$ can be exhausted by $D$ or $D$ can exhaust $\Omega$ if for any compact subset $K$ of $\Omega$ there exists an injective holomorphic map $f: D \rightarrow \Omega$ such that $K \subset f(D)$.

It is proved by Fridman that there are domains in $\mathbb{C}^{m}$ which can exhaust all domains [18. It is also observed by Fornæss that the unit ball $B^{n}$ can exhaust many domains which are not biholomorphic to each other [12. However, if we restrict on bounded domains, things become very different. For example, it is easy to prove that if a bounded domain $\Omega$ can be exhausted by a homogenous domain $D$, then $\Omega$ must be holomorphic equivalent to $D$ (see [18]).

In [18, Fridman shows that if a bounded domain $\Omega$ can be exhausted by a bounded strictly pseudoconvex domain $D$ with $C^{3}$ boundary, then $\Omega$ must be biholomorphic to $D$ or the unit ball $B^{n}$. The proof is based on the boundary estimate of $h_{D}$ for strictly pseudoconvex domains with $C^{3}$ bounday. In this note, applying the estimate of squeezing functions in [7] and based on Firdman's idea, we show that the same result still holds if the boundary regularity of $D$ is reduced to $C^{2}$.

Theorem 1.1. Let $D$ be a bounded strictly pseudoconvex domain in $\mathbb{C}^{n}$ with $C^{2}$ boundary. If a bounded domain $\Omega \subset \mathbb{C}^{n}$ can be exhausted by $D$, then $\Omega$ must be biholomorphic to $D$ or the unit ball $B^{n}$.

Another result in this note about exhausting domains is the following

Theorem 1.2. If a bounded domain $\Omega$ can be exhausted by a homogenous regular domain, then $\Omega$ is homogenous regular.

Another purpose of the present note is to give an boundary estimate of $h_{D}$. By comparing with squeezing functions, we consider a variant $e_{D}$ of $h_{D}$ which is defined by

$$
h_{D}^{-1}(z)=\log \frac{1+e_{D}(z)}{1-e_{D}(z)} .
$$

Then $e_{D}(z) \in(0,1]$ and is set to be 1 if $h_{D}(z)=0$.

Theorem 1.3. Let $D \subset \mathbb{C}^{n}$ be a bounded domain and $p \in \partial D$ be a $C^{2}$ boundary point of $D$. If there is a sequence $z_{j} \in D(j \geq 1)$ converging to $p$ and a sequence of positive numbers $\epsilon_{j}(j \geq 1)$ converges to 0 such that $e_{D}\left(z_{j}\right)>1-\epsilon_{j} \delta\left(z_{j}\right)$ for all $j$, then $D$ is biholomorphic to the unit ball, where $\delta(z)$ denotes the distance between $z$ and $\partial D$.

By the decreasing property of the Kobayashi metric, it is obvious that $s_{D} \leq e_{D}$ for all bounded domains (see 28]). So as a corollary of Theorem 1.1] we get the main result of Diederich-Fornæss-Wold in 10] which says that, for a bounded strictly pseudocovnex domain $D$ with $C^{2}$ boundary which is not biholomorphic to the unit ball, $s_{D}(z) \leq C \delta(z)$ for some constant $C$. 
On the other hand, it is proved by Fornæss-Wold that for a bounded strictly pseudoconvex domain $D$ with $C^{4}$ boundary, the estimate

$$
s_{D}(z) \geq 1-C \delta(z), z \in D
$$

holds for some constant $C>0$ [16]. Combing this result with Theorem [1.3, we obtain the following

Corollary 1.4. Let $D$ be a bounded strictly pseudoconvex domain with $C^{4}$ boundary. Then there exists a constant $C>0$ such that

$$
1-C^{-1} \delta(z) \leq e_{D}(z) \leq 1-C \delta(z), z \in D .
$$

It seems that for a strictly pseudoconvex domain $D$, the boundary behaviour of $e_{D}(z)$ and $s_{D}(z)$ are very similar. So we conjecture that the following comparison

$$
\lim _{z \rightarrow \partial D} \frac{1-e_{D}(z)}{1-s_{D}(z)}=1
$$

holds.

During the preparation of this note, a paper of Nikolov and Verma 28 discussing some related topics appears in arXiv. After the first version of this note appeared in arXiv, Professor Nikolai Nikolov pointed out to the authors that Theorem 1.3 in this note is contained in Proposition 8 in 28. Proposition 8 in 28] is stated for squeezing functions and itself can not contain Theorem 1.3. But after looking into the details of its proof, we realized that the same argument can give a proof of Theorem 1.3 .

Acknowledgements. The first author thanks Professor John Erik Fornæss for discussions. The authors are partially supported by NSFC grants.

\section{Exhausting A DOMAin BY STRICTLY PSEUdoconVEX DOMAins}

The aim of this section if to prove Theorem 1.1.

We first recall some results about squeezing functions from [6] [7].

Lemma 2.1 ([6]). Let $D \subset \mathbb{C}^{n}$ be a bounded domain. If there is a point $z \in D$ such that $s_{D}(z)=1$, then $D$ is biholomorphic to the unit ball $\mathbb{B}^{n}$.

Lemma $2.2(77)$. Let $D, D_{k} \subset \mathbb{C}^{n}(k \geq 1)$ are bounded domains with $D_{k} \subset D$ such that, for any compact set $K \subset D$, there is $N>0$ such that $K \subset D_{k}$ for all $k>N$. Then for any $z \in D$, we have $\lim _{k \rightarrow \infty} s_{D_{k}}(z)=s_{D}(z)$.

In [7, the domains $D_{k}$ are required to be increasing, but the same proof can give the result in the above lemma.

Lemma 2.3 ([7). Let $D \subset \mathbb{C}^{m}$ be a bounded domain such that the closure $\bar{D}$ of $D$ admits a Stein open neighborhood basis. Then for any $C^{2}$ strictly pseudoconvex boundary point $p$ of $D$, we have $\lim _{z \rightarrow p} s_{D}(z)=1$.

We can now give the proof of Theorem 1.1. For convenience, we restate it here.

Theorem 2.4. Let $D$ be a bounded strictly pseudoconvex domain in $\mathbb{C}^{n}$ with $C^{2}$ boundary. If a bounded domain $\Omega \subset \mathbb{C}^{n}$ can be exhausted by $D$, then $\Omega$ must be biholomorphic to $D$ or the unit ball $B^{n}$. 
Proof. Let $f_{k}: D \rightarrow \Omega(k \geq 1)$ be a sequence of holomorphic injective maps satisfying, for any compact set $K \subset \Omega$, there is $N>0$ such that $K \subset f_{k}(D)$ for all $k>N$.

Fix a point $w_{0} \in \Omega$, we consider the inverse images $\left\{z_{k}:=f_{k}^{-1}\left(w_{0}\right) ; k \geq 1\right\}$. We first assume that the set has an accumulation point $z_{0} \in D$. Without loss of generality, we may assume $\lim _{k \rightarrow \infty} z_{k}=z_{0}$. Let $g_{k}:=f_{k}^{-1}: f_{k}(D) \rightarrow D$. By Montel theorem, we may assume $f_{k}$ converges to $f: D \rightarrow \mathbb{C}^{n}$ and $g_{k}$ converges to $g: \Omega \rightarrow \mathbb{C}^{n}$ uniformly on compact sets. We want to show that $f(D) \subset \Omega$ and $g(\Omega) \subset D$ and $f \circ g=g \circ f=I d$. By assumption, we have $f_{k}\left(z_{k}\right) \rightarrow w_{0}=f\left(z_{0}\right)$ as $k \rightarrow \infty$. By Cauchy inequality, we see $J_{g}\left(w_{0}\right) \neq 0$ and $J_{f}\left(z_{0}\right) \neq 0$, where $J_{f}$ and $J_{g}$ are the Jacobian of $f$ and $g$. By Rouché's theorem, $J_{f} \neq 0$ and $J_{g} \neq 0$ everywhere. In particular, $f$ and $g$ are locally biholomorphic. By the generalized Ruché's theorem for holomorphic maps (see 24]), we have $f(D) \subset \Omega$ and $g(\Omega) \subset D$. Then the equalities $f \circ g=I d_{\Omega}$ and $g \circ f=I d_{D}$ follows obviously. Therefore, in this case, we get that $\Omega$ is biholomorphic to $D$.

We now assume that $\left\{z_{k}:=f_{k}^{-1}\left(w_{0}\right) ; k \geq 1\right\}$ has no accumulation point inside $D$. Then we may assume that $\lim _{k \rightarrow \infty} z_{k}=p$ for some $p \in \partial D$. By biholomorphic invariance, we have $s_{D}\left(z_{k}\right)=s_{f_{k}(D)}\left(w_{0}\right)$ for all $k$. By Lemma 2.2. we have $\lim _{k} s_{f_{k}(D)}\left(w_{0}\right)=s_{\Omega}\left(w_{0}\right)$. By Lemma 2.3 we have $\lim _{k} s_{D}\left(z_{k}\right)=1$ and hence $s_{\Omega}\left(w_{0}\right)=1$. By Lemma 2.1, $\Omega$ is biholomorphic to the unit ball.

\section{Exhausting DOMAins By homogenous REgular DOMAins}

In this section we give the proof of Theorem 1.2 which says that a domain is homogenous regular if it can be exhausted by a homogenous regular domain.

Recall that a bounded domain $D$ is called homogenous regular if the squeezing function $s_{D}$ of $D$ has a positive lower bound.

We now give the proof of Theorem Theorem 1.2 .

Proof of Theorem Theorem 1.2. Let $D$ be a homogenous regular domain. We assume that $s_{D} \geq c$ for some constant $c>0$. Assume that $\Omega$ is a bounded domain that can be exhausted by $D$. We want to show that $s_{\Omega} \geq c$ and hence $\Omega$ is also homogenous regular.

Let $f_{k}: D \rightarrow \Omega$ be holomorphic injective maps such that for any compact set $K \subset \Omega$, there is $N>0$ such that $K \subset f_{k}(D)$ for all $k \geq N$.

Let $w \in \Omega$ and set $z_{k}=f^{-1}(w)$. By Lemma 2.2, $s_{\Omega}(w)=\lim _{k} s_{f_{k}(D)}\left(z_{k}\right)$. By invariance of squeezing functions under biholomorphic transformations, we have $s_{f_{k}(D)}\left(z_{k}\right)=s_{D}\left(z_{k}\right)$ and hence $s_{f_{k}(D)}\left(z_{k}\right) \geq c$ for all $k$. Hence $s_{\Omega}(w) \geq c$. Since $w$ is arbitrary, $s_{\Omega} \geq c$ and hence $\Omega$ is also homogenous regular.

\section{Boundary ESTIMATES OF FRIDMAN's INVARIANTS}

This section is to prove Theorem 1.3

We first give an estimate of upper bound of the Kobayashi distance of a bounded domain near a $C^{2}$ boundary point.

Lemma 4.1. Assume $D \subset \mathbb{C}^{n}$ is a bounded domain with $z_{0} \in D$. Let $p \in \partial D$ be a $C^{2}$ boundary point of $D$. Let $d_{D}(\cdot, \cdot)$ be the Kobayashi distance on $D$. Then there exists a constant $C$ such that

$$
d_{D}\left(z_{0}, z\right) \leq \log \frac{1}{\delta(z)}+C
$$


for $p$ in some small neighborhood of $p$, where $\delta(z)$ is the Euclidean distance from $z$ to $\partial D$.

Proof. By some basic results from differential topology, there is an open neighborhood $U$ such that for any $z \in U \cap D$ there is a unique point $\pi(z) \in U \cap \partial D$ such that $\delta(z)=d(z, \pi(z))$. Let $r>0$ be sufficiently small and let $M:=\{z \in \bar{V} \cap D ; \delta(z)=$ $r\}$, where $V \subset U$ is an open neighborhood of $p$ such that $\bar{V} \subset U$. Then for any $z \in M$, the 1-dimensional complex disc $\Delta_{z}$ in the direction $\pi(z)-z$ centered at $z$ and with radius $r$ is contained in $D$.

Now for any $z \in D$ which is sufficiently close to $p$, we can find a unique $a \in M$ such that $z \in \Delta_{a}$. By the triangle inequality and decreasing property for Kobayashi distance, we have

$$
d_{D}\left(z_{0}, z\right) \leq d_{D}\left(z_{0}, a\right)+d_{\Delta_{a}}(a, z)=d_{D}\left(z_{0}, a\right)+\log \frac{2 r-\delta(z)}{\delta(z)} .
$$

Note that $M$ is compact and $r$ is independent of $z, D\left(z_{0}, z\right) \leq C+\log \frac{1}{\delta(z)}$ for some constant $C>0$.

We now give the proof of Theorem 1.3 For convenience, we restate it here.

Theorem 4.2. Let $D \subset \mathbb{C}^{n}$ be a bounded domain and $p \in \partial D$ be a $C^{2}$ boundary point of $D$. If there is a sequence $z_{j} \in D(j \geq 1)$ converging to $p$ and a sequence of positive numbers $\epsilon_{j}(j \geq 1)$ converges to 0 such that $e_{D}\left(z_{j}\right)>1-\epsilon_{j} \delta\left(z_{j}\right)$ for all $j$, then $D$ is biholomorphic to the unit ball, where $\delta(z)$ denotes the distance between $z$ and $\partial D$.

Proof. We will show that $D$ can be exhausted by the unit ball $B^{n}$.

Let $s_{j}=1-\epsilon_{j} \delta\left(z_{j}\right)$ and let

$$
r_{j}=\log \frac{1+s_{j}}{1-s_{j}}=\log \frac{2+\epsilon_{j} \delta\left(z_{j}\right)}{\epsilon_{j} \delta\left(z_{j}\right)} .
$$

By definition of $e_{D}(z)$, for each $j$, there is a holomorphic injective map $f_{j}: B^{n} \rightarrow D$ such that $f_{j}(0)=z_{j}$ and $B_{D}^{K}\left(z_{j}, r_{j}\right) \subset f_{j}\left(B^{n}\right)$, where $B_{D}^{K}\left(z_{j}, r_{j}\right)$ is the ball with respect to the Kobayashi metric in $D$ with center $z_{j}$ and radius $r_{j}$.

Let $A$ be an arbitrary compact set in $D$. By Lemma 4.1 and the continuity of Kobayashi metric, there is a constant $C>0$ independent of $z_{j}$ such that $d_{D}\left(A, z_{j}\right) \leq$ $\log \frac{1}{\delta\left(z_{j}\right)}+C$ for $j$ sufficiently large, where $d_{D}(\cdot, \cdot)$ is the Kobayashi distance on $D$. This implies that $A \subset B_{D}^{K}\left(z_{j}, r_{j}\right) \subset f_{j}\left(B^{n}\right)$ for $j$ large enough. Therefore $\Omega$ can exhausted by the unit ball $B^{n}$. By Theorem 1.1, $D$ is biholomoprhic to $B^{n}$.

\section{REFERENCES}

[1] K. Diederich, J. E. Fornæss, E. F. Wold, A characterization of the ball, preprint, arXiv:1604.05057

[2] E. Andersén and L. Lempert, On the group of holomorphic automorphisms of $\mathbb{C}^{n}$, Invent. Math. 110 (1992), 371-388.

[3] L. Arosio, J. E. Foræss, N. Shcherbina, E. F. Wold, Squeezing functions and Cantor Sets, e-print, arXiv: 1710.10305.

[4] F. Bracci, J. E. Foræss, E. F. Wold, Comparison of invariant metrics and distances on strongly pseudoconvex domains and worm domains, e-print, arXiv:1710.04192

[5] F. Deng, J. E. Fornæss, E. F. Wold, Exposing boundary points of strongly pseudoconvex subvarieties in complex spaces, e-print, arXiv:1607.02755 to appear in Proc. Amer. Math. Soc.. 
[6] F. Deng, Q. Guan, L. Zhang, On some properties of squeezing functions on bounded domains, Pacific J. Math. V. 57, No.2 (2012), 319-342.

[7] F. Deng, Q. Guan, L. Zhang, Properties of squeezing functions and global transformations of bounded domains, Transactions of AMS, 368 (2016), 2679-2696.

[8] K. Diederich, J.E. Fornæss, Boundary Behavior of the Bergman Metric, e-preprint, arXiv:1504.02950

[9] K. Diederich, J.E. Fornæss, Pseudoconvex domains: An example with nontrivial Nebenhülle, Math. Ann. 225(1977), 275-292.

[10] K. Diederich, J.E. Fornæss, E.F. Wold, Exposing points on the boundary of a strictly pseudoconvex or a locally convexifiable domain of finite 1-type, Journal of Geometric Analysis, 24 (2014), 2124-2134.

[11] K. Diederich, J.E. Fornæss, E.F. Wold, A characterization of the ball in $\mathbb{C}^{n}$, International Journal of Mathematics, 27 (2016).

[12] J. E. Fornæss, Short $\mathbb{C}^{k}$, Adv. Stud. Pure Math., 42, Math. Soc. Japan, Tokyo, 2004. 32E10 (32E40).

[13] J.E. Foræss, K.T. Kim, Some problems, Springer Proceedings in Mathematics \& statistics, 144(2015), 369-377.

[14] J. E. Foræss, F. Rong, Estimate of the squeezing function for a class of bounded domains, e-preprint, arXiv:1606.01335 to appear in Math. Ann..

[15] J. E. Foræss, N. Shcherbina, A domain with non-plurisubharmonic squeezing function, epreprint, arXiv:1604.01480

[16] J.E. Fornæss, E.F. Wold, An estimate for the squeezing function and estimates of invariant metrics. Complex Analysis and Geometry, Springer Proceedings in Mathematics \& statistics, 144(2015), 135- 147.

[17] J.E. Fornæss, E.F. Wold, A non-strictly pseudoconvex domain for which the squeezing function tends to one towards the boundary, e-preprint, arXiv:1611.04464

[18] B. L. Fridman, Biholomorphic invariants of a hyperbolic manifold and some application, Trans.Amer.Math.Soc., 276, 685-698 (1983).

[19] B. L. Fridman, A universal exhausting domain, Proc. Amer. Math. Soc. 98 (1986), no. 2, $267 \mathrm{C} 270$.

[20] S. Joo, K.-T. Kim, On boundary points at which the squeezing function tends to one, e-print, arXiv:1611.08356

[21] K.-T. Kim, L. Zhang, On the uniform squeezing property and the squeezing function, Pacific J. Math. 282 (2016), 341-358.

[22] K. Liu, X. Sun, S.T. Yau, Canonical metrics on the moduli space of Riemann Surfaces I, J. Differential Geom. Vol. 68 (2004), 571-637.

[23] K. Liu, X. Sun, S.T. Yau, Canonical metrics on the moduli space of Riemann Surfaces II , J. Differential Geom. Vol. 69 (2005), 163-216.

[24] N. G. Lloyd. Remarks on generalising Rouché's theorem. J. London Math. Soc. (2), 20(2):259$272,1979$.

[25] N. Nikolov, Behavior of the squeezing function near h-extendible boundary points, Proc. Amer. Math. Soc. 146 (2018), No 8, 3455-3457.

[26] N. Nikolov, L. Andreev, Boundary behavior of the squeezing functions of complex domains,Int. J. Math. 28 (2017), No 5.

[27] N. Nikolov, M. Trybula, Estimates for the squeezing function near strictly pseudoconvex boundary points with applications, arXiv:1808.07892

[28] N. Nikolov, K. Verma, On the squeezing function and Fridman invariants, e-preprint, arXiv: 181010739 .

[29] S. K. Yeung, Geometry of domains with the uniform squeezing property, Adv. Math. 221 (2009) 547-569.

[30] L. Zhang, On curvature estimates of bounded domains, Complex Analysis and Geometry, Springer Proceedings in Mathematics \& statistics, 144(2015), 353-367.

[31] A. Zimmer, A gap theorem for the complex geometry of convex domains, e-preprint, arXiv:1609.07050, to appear in Transactions of the AMS.

[32] A. Zimmer, Characterizing strong pseudoconvexity, obstructions to biholomorphisms, and Lyapunov exponents, e-preprint, arXiv:1703.01511.

[33] A. Zimmer, Smoothly bounded domains covering finite volume manifolds, e-preprint, arXiv:1802.01178 
Fusheng Deng: School of Mathematical Sciences, University of Chinese Academy of Sciences, Beijing 100049, China

E-mail address: fshdeng@ucas.ac.cn

Xujun Zhang: School of Mathematical Sciences, University of Chinese Academy of Sciences, Beijing 100049, China

E-mail address: zhangxujunmath@icloud.com 